\title{
Septic Shock Due to Candidemia: Outcomes and Predictors of Shock Development
}

\author{
Jorge A Guzman ${ }^{\mathrm{a}, \mathrm{d}}$, Ronny Tchokonte ${ }^{\mathrm{b}}$, Jack D Sobel ${ }^{\mathrm{c}}$
}

\begin{abstract}
Background: The present report describes the outcomes of a cohort of patients with Candida induced septic shock.

Methods: Retrospective analysis of individuals who had at least one positive blood culture for Candida species $\geq 48 \mathrm{~h}$ after ICU admission. Data from patients that developed septic shock within $48 \mathrm{hr}$ of the positive blood culture were compared to non-shock candidemic patients. Patients with a concomitant bacteremia and/or endocarditis were excluded.
\end{abstract}

Results: Fifteen patients with Candida induced septic shock were studied and compared to 35 candidemic patients without shock. Overall mortality was $76 \%$ ( $87 \%$ among those who had shock). A high proportion of non-albicans Candida species causing fungemia (74\%) was observed. All patients with shock were receiving antibiotics but not antifungal treatment at the time of shock development, eight were on parenteral nutrition, six on steroids and nine had a cancer history. High dose fluconazole was the most common initial treatment provided. Four patients died before receiving any antifungal treatment. Time in ICU before the development of candidemia was identified as a predictor of shock development (higher chance if fungemia developed $<7$ days after ICU admission).

Conclusions: Septic shock due to invasive candidiasis is a near fatal condition. No conventional risk factors were identified to predict shock development other than time (shorter) spent in ICU before the development of candidemia. We encourage clinicians to consider the initiation of appropriate empiric antifungal treatment in

Manuscript accepted for publication March 10, 2011

${ }^{a}$ Section of Critical Care, Respiratory Institute, Cleveland Clinic, Cleveland, USA

${ }^{\mathrm{b}}$ Pulmonary and Critical Care, Wayne State University, Detroit, USA

${ }^{\mathrm{c}}$ Division of Infectious Diseases, Wayne State University, Detroit, USA

${ }^{\mathrm{d} C}$ Corresponding author: Jorge A Guzman, 9500 Euclid Ave, G6-156,

Cleveland OH 44195, USA. Email: guzmanj@ccf.org

doi:10.4021/jocmr536w high-risk patients who develop septic shock while on antimicrobial treatment.

Keywords: Septic shock; Candidemia; Outcome; Predictor

\section{Introduction}

Candida is an increasing cause of bloodstream infection, causing significant mortality and morbidity. Its overall incidence rose fivefold in the past ten years and remains the fourth leading cause of nosocomial bloodstream infection in the United States, accounting for 8\% of all bloodstream infections acquired in hospitals [1-3]. Furthermore, a change in the epidemiology has also been noted. Species other than $C$ albicans have increased and surpassed $C$ albicans in incidence in some tertiary care centers [4-7]. Some of these emerging species have been correlated with increased virulence [8], and in some reports with increased mortality [9]. Despite the availability of effective antifungal therapy, crude mortality in the last decade has remained high, ranging from 36 to $90 \%$ [9-11].

A number of predisposing factors have been associated with development of invasive candidiasis and clinical predictors of poor outcome have been reported [12-14]. Nevertheless, studies describing the clinical course of patients developing candidemia are scant [15], and limited data exist of the outcomes of patients who develop septic shock due to invasive candidiasis [16]. In general, when patients develop septic shock, bacterial causes are exclusively considered and no effort to empirically treat Candida species is made.

The present report describes a cohort of patients with Candida induced septic shock. In order to identify predictors of shock development, data obtained from patients in shock were compared to a group of patients who had ICU acquired candidemia without shock.

\section{Materials and Methods}

The Human Investigation Committee (HIC) of Wayne State 


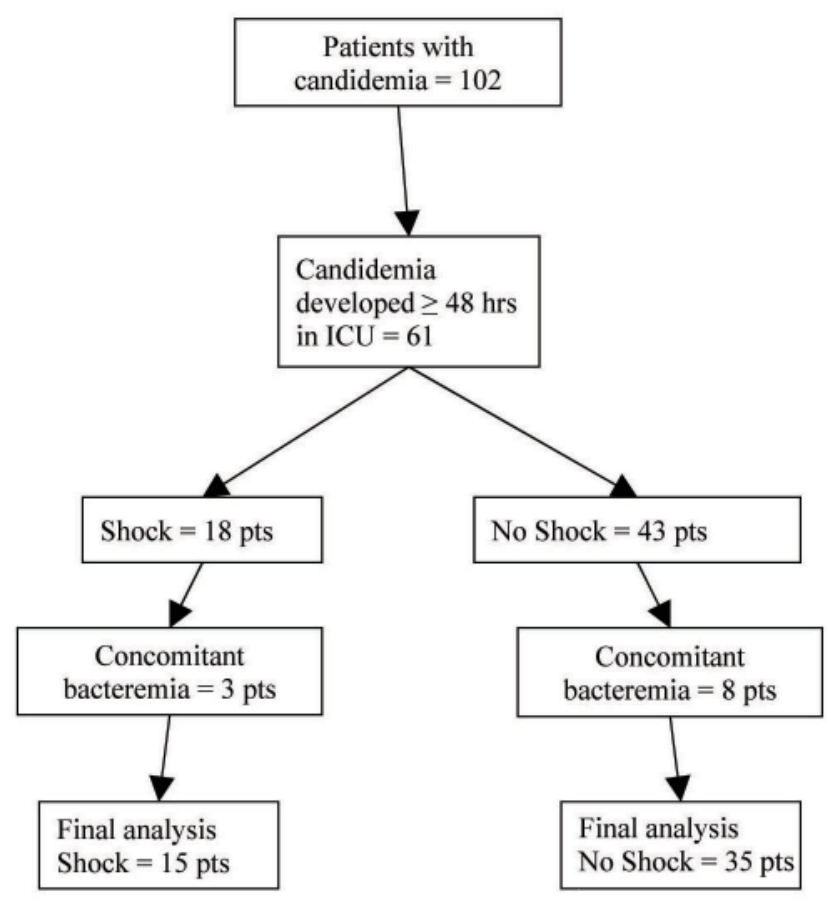

Figure 1. Flowchart of study subjects.

University approved the study protocol. The medical records of patients who had at least one positive blood culture specimen of Candida spp. collected after their ICU admission at Harper University Hospital over a five year span (1998 2003) were reviewed. Case patients were defined as individuals who had at least one positive blood culture for Candida species grown $\geq 48 \mathrm{~h}$ after ICU admission and in whom septic shock developed within $48 \mathrm{hrs}$ of a positive blood culture. Patients who had a blood culture positive for a concomitant bacterial pathogen (i.e., mixed infection) and patients with endocarditis were excluded.

\section{Definitions}

Septic shock: acute circulatory failure characterized by persistent arterial hypotension (systolic arterial pressure below $90 \mathrm{~mm} \mathrm{Hg}$, a MAP $<60 \mathrm{~mm} \mathrm{Hg}$, or a reduction in systolic blood pressure of $>40 \mathrm{~mm} \mathrm{Hg}$ from baseline, despite adequate volume resuscitation, or the need for vasopressors in the absence of other causes for hypotension) in the presence of candidemia [17]. ICU acquired candidemia: Positive blood culture for Candida spp. grown $\geq 48 \mathrm{hrs}$ after ICU admission. Colonization was defined as presence of Candida spp. in a non-sterile site. Candiduria as the presence of $\geq 10^{4}$ $\mathrm{cfu} / \mathrm{mL}$ of Candida spp. in urine. Airway colonization: Candida spp. isolated from tracheal aspirates or bronchoalveolar lavage specimens. Catheter-associated candidemia was de- termined as $\geq 15$ cfu of Candida spp. isolated from a central venous catheter tip.

\section{Study variables}

Study population characteristics and data extracted included demographics, age, and sex of the patients, reason for ICU admission, APACHE (Acute Physiology and Chronic Health Evaluation) II score at admission, length of stay (LOS) in ICU, hospital LOS, LOS prior to ICU admission, time in ICU before development of candidemia, predisposing risk factors for candidemia such as diabetes mellitus, broad spectrum antibiotic treatment (for $>3$ days) during the hospital stay, chronic renal failure, liver disease, Candida colonization, systematic administration of glucocorticosteroids for any reason in the last 30 days before ICU admission (regardless of dose), total parenteral nutrition administration, central venous catheter (CVC) placement (for $>2$ days), the day of candidemia occurrence, and outcome (i.e., dead or alive at ICU discharge).

\section{Statistical analysis}

The $[\mathrm{chi}]^{2}$ test or Fisher's exact test was used to evaluate categorical variables and the Student's $t$-test to evaluate continuous variables. Multiple logistic regression analysis was performed to identify independent variables associated with 


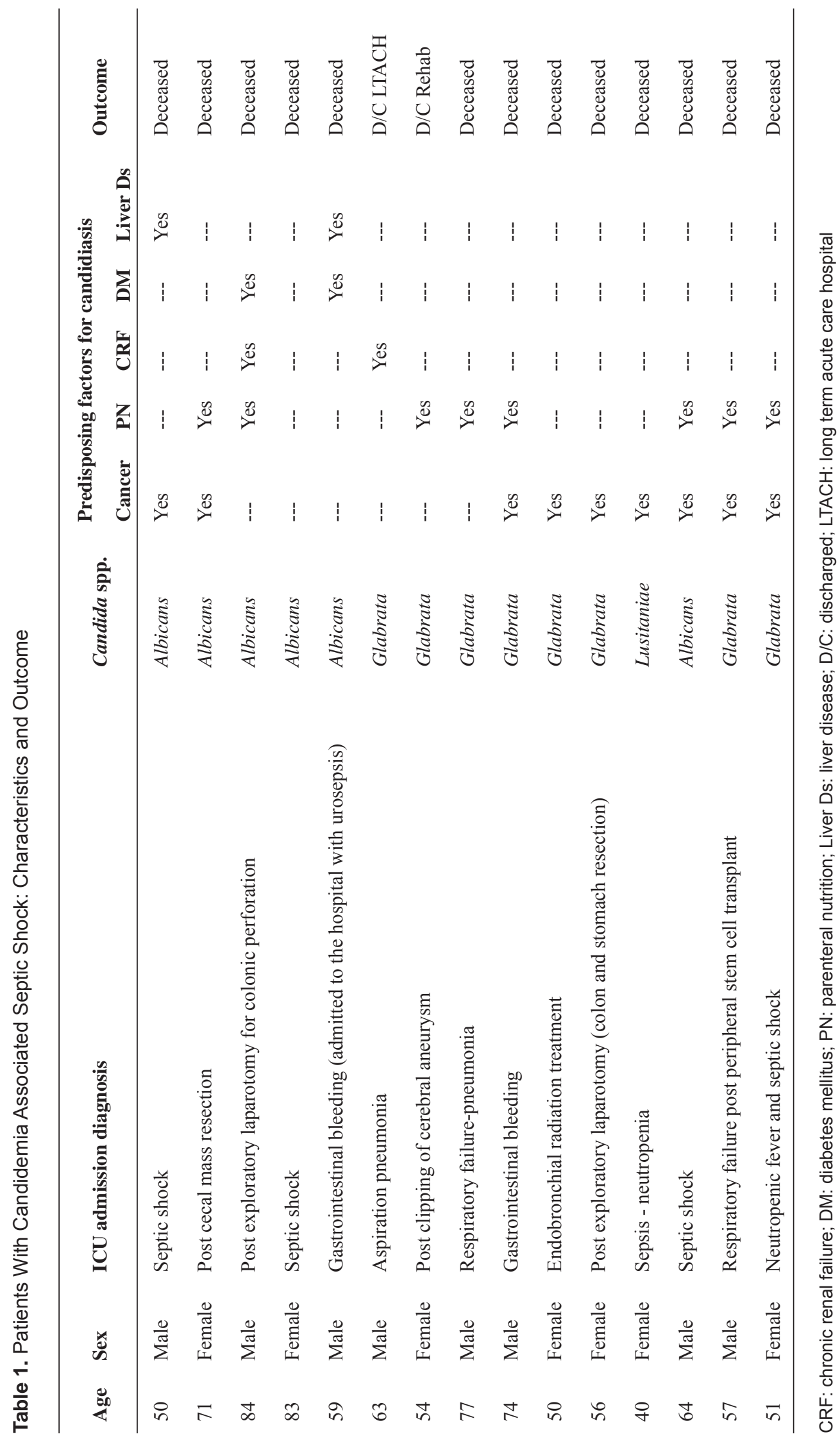


Table 2. Patient Age, Acuity, Outcomes and Lengths of Stay

\begin{tabular}{lllll}
\hline & $\begin{array}{l}\text { All Candidemias } \\
(\mathbf{n}=\mathbf{5 0})\end{array}$ & $\begin{array}{l}\text { No shock } \\
(\mathbf{n}=\mathbf{3 5})\end{array}$ & $\begin{array}{l}\text { Shock } \\
(\mathbf{n}=\mathbf{1 5})\end{array}$ & P Value \\
\hline Age (years) & $61 \pm 14$ & $60 \pm 14$ & $62 \pm 13$ & 0.64 \\
Apache II & $22.6 \pm 8.3$ & $21.1 \pm 7.2$ & $26.5 \pm 9.7$ & 0.04 \\
Mortality (\%) & 76 & 71 & 87 & 0.30 \\
Hospital LOS (days) & $35.6 \pm 19.8$ & $40.1 \pm 19.1$ & $25.1 \pm 17.9$ & 0.01 \\
ICU LOS (days) & $22.0 \pm 16.0$ & $24.0 \pm 16.5$ & $17.4 \pm 14.3$ & 0.18 \\
Hospital LOS prior to ICU (days) & $7.9 \pm 10.5$ & $9.3 \pm 11.5$ & $4.4 \pm 6.7$ & 0.13 \\
Time in ICU prior to candidemia & $13.5 \pm 13.2$ & $16.9 \pm 14.4$ & $5.5 \pm 3.8$ & 0.004 \\
Documented colonization (patients) & $22(44 \%)$ & $18(51 \%)$ & $4(27 \%)$ & 0.13 \\
Candida spp. patients (\%) & & & & \\
Albicans & $14(26 \%)$ & $8(23 \%)$ & $6(40 \%)$ & 0.30 \\
Non-albicans & $36(74 \%)$ & $27(77 \%)$ & $9(60 \%)$ & \\
Time to clearance (days) & $8.6 \pm 8.3$ & $7.4 \pm 7.9$ & $9.0 \pm 8.6$ & 0.64 \\
\hline
\end{tabular}

LOS: Length of stay

development of shock. Potential risk factors were included in the analysis if they were associated with the dependent variables in the univariate analyses at a statistical level $\mathrm{P}<$ 0.2 . A $\mathrm{P}<0.05$ was considered statistically significant in the multivariable models to show association between various potential risk factors and the dependent variable. An empirical receiver operating curve (ROC) was created for each predictor. Also, sensitivity and specificity for each predictor were calculated. The optimal threshold was chosen in such a way that the threshold made the resulting binary prediction as close to the perfect operating point as possible. The Euclidean method was used to measure the distance between an observed point on the ROC curve and the perfect point.

\section{Results}

Fifteen patients with Candida induced septic shock were identified and included in the study. Data obtained from this group was then compared to thirty-five patients with ICU acquired candidemia who did not develop shock (Fig. 1). The main characteristics of patients who developed shock are shown in Table 1. Patients with shock had higher illness acuity, most had a history of cancer $(60 \%)$ and four were neutropenic at the time of ICU admission. Candidemia in patients with shock developed $5.5 \pm 3.8$ days after ICU admission; only three patients had a positive blood culture within $48 \mathrm{hrs}$ of ICU admission. Mortality among patients with Candida induced septic shock was $87 \%$. All patients were receiving antibiotic treatment (mean number of antibiotic agents 4.1 \pm 1.7 ) for $4.2 \pm 3.9$ days prior to the development of candidemia. Six $(40 \%)$ patients were on corticosteroids (mean duration of treatment prior to candidemia $4.9 \pm 2.6$ days) and eight $(53 \%)$ were on parenteral alimentation at the time of development of candidemia. All patients had at least one CVC in place. Ten patients were initially treated with high dose fluconazole ( $\geq 12 \mathrm{mg} / \mathrm{kg} / \mathrm{day})$, one with amphotericin $\mathrm{B}$, and four patients died before they received any antifungal treatment (culture results positive post mortem). Eight of the eleven treated patients cleared the fungemia after $7.4 \pm 7.9$ days of antifungal treatment, and 3 patients had persistent candidemia. Antifungal therapy was subsequently changed in 6 patients (five placed on deoxycholate amphotericin B and 1 on voriconazole).

Table 2 shows data for the whole cohort and compares candidemic patients with and without shock. Overall mortality was very high (76\%). Patients in shock had a higher illness acuity and higher mortality. ICU stay prior to the development of candidemia was shorter in patients who developed shock and so was their overall hospital LOS (presumably because these patients died more rapidly). Duration of candidemia was identical in both groups. Although there were proportionally more patients with Candida albicans who developed shock, no significant statistical difference in species was noted between the two groups. Four patients in the shock group (27\%) had documented Candida colonization (three patients had combined urine and sputum, one patient had only sputum 
Table 3. Multiple Logistic Regression Analysis

\begin{tabular}{llll}
\hline & Odds ratio & $\mathbf{9 5 \%}$ CI & P Value \\
\hline APACHE II score & 1.007 & $0.882-1.149$ & 0.919 \\
Hospital LOS & 0.902 & $0.805-1.010$ & 0.075 \\
ICU LOS & 1.119 & $0.988-1.267$ & 0.076 \\
Hospital LOS before ICU & 0.965 & $0.819-1.138$ & 0.675 \\
ICU LOS prior to candidemia & 0.714 & $0.531-0.960$ & 0.026 \\
Candida colonization & 0.291 & $0.037-2.258$ & 0.237 \\
\hline
\end{tabular}

Hosmer-Lemeshow statistic: $3.175(P=0.923)$; Likelihood ratio test statistic: $28.474(P \leq 0.001)$ ICU: Intensive care unit; APACHE: Acute Physiology and Chronic Health Evaluation; LOS: Length of stay

colonization), whereas eighteen patients $(51 \%)$ in the noshock group were colonized (eight patients urine, 10 sputum and 5 patients catheter-related). The difference did not reach statistical significance $(\mathrm{P}=0.135)$.

Table 3 shows odd ratios and confidence intervals for shock development for the parameters identified by univariate analysis. Time in ICU before the development of candidemia reached significance as predictor of shock development. Area under the receiver operating curve was 0.826 (Fig. 2). The threshold of $<7.2$ days in ICU before the development of candidemia allowed a discrimination between shock development and no shock with a sensitivity of $73.3 \%$ and a specificity of $80.0 \%$. A trend was also noted for hospital and ICU LOS prior to development of candidemia, the lack of statistical significance may be related to the limited sample size. Judging by the values of the Hosmer-Lemeshow and the likelihood ratio statistic, the model appears well calibrated.

\section{Discussion}

The present study shows that Candida induced septic shock is a nearly fatal condition with mortality rate that triples that of bacterial septic shock. Estimation of the true incidence of Candida induced septic shock is not possible from this study, although the percentage of patients with shock i.e. $30 \%$ in our ICU cohort is similar to the $27 \%$ and $23 \%$ frequency pre-

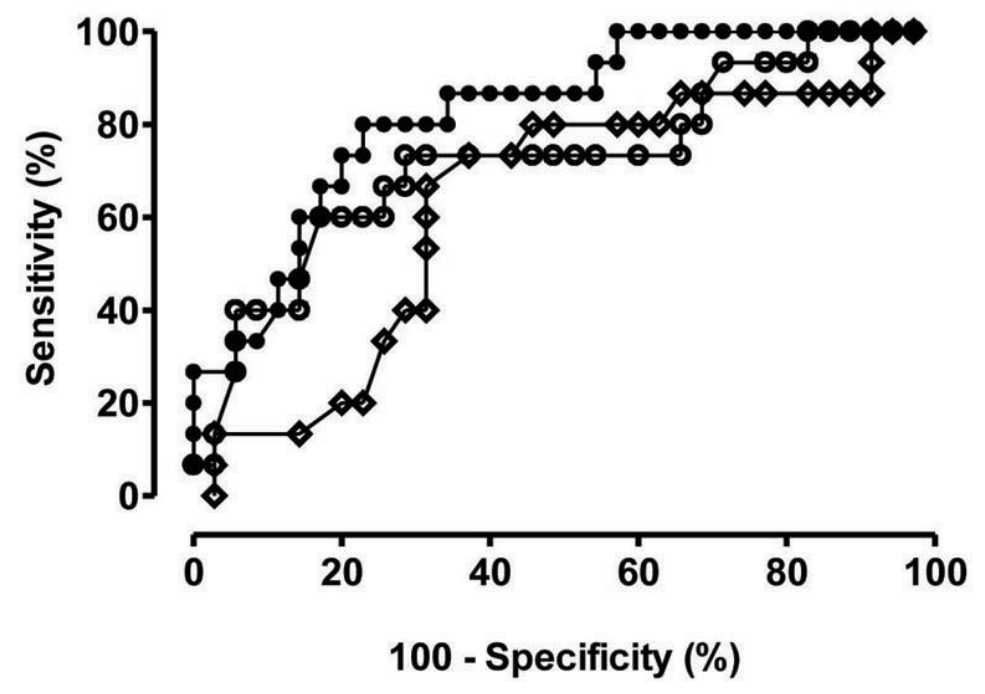

Figure 2. Figure 2. Receiver operator curves for the three predictors with statistical significance. Closed circles: ROC for time in ICU prior to candidemia; Open circles: ROC for ICU length of stay (LOS); Open diamonds: ROC for hospital LOS 
viously reported $[15,18]$. The comparable rates strengthen the selection criteria employed to identify case patients in this retrospective cohort and highlight the high risk of progression to septic shock among critically ill patients who develop candidemia, a fact ignored by many clinicians.

The high mortality observed among ICU patients with candidemia has been attributed to a number of factors, including APACHE scores, inadequate initial fluconazole dosing, delayed administration of antifungal therapy due to slow fungal growth or insensitive culture methods, or retention of central venous catheters $[12,18]$. High dose fluconazole was the initial treatment in all but one patient. Although current treatment guidelines suggest use of echinocandin agents or polyenes in hemodynamically unstable patient [19], these recommendations were published after the study occurred (1988 - 2003) and before the availability of echinocandins. It remains speculative whether the outcomes observed in this cohort have any relationship to the treatment provided. Nevertheless, it is still feasible that the high mortality observed was a consequence of using a fungistatic agent such as fluconazole rather than fungicidal echinocandin or polyene agents. A more likely explanation is the time that our patients were without antifungal treatment awaiting blood cultures to turn positive. This emphasizes the need to introduce sensitive diagnostic assays that facilitate earlier pathogen identification. Supporting this concept is the fact that almost a third of our patients died before isolating the organism responsible for septic shock and therefore did not receive antifungal treatment. All patients were being treated with multiple antibiotic agents at the time of shock development, begging the question whether clinicians should consider empiric antifungal therapy in patients who develop shock while receiving broad spectrum antimicrobial agents.

Although a number of studies have described risk factors for development of candidemia, none have specifically identified factors predisposing to development of shock [13, $14,20,21]$. Using a multiple regression analysis we were able to identify the time spent in ICU until the development of candidemia as the only parameter that reached statistical significance as shock predictor. ROC analysis suggests using a threshold of 7 days in the unit as the best way to identify who may develop shock due to candidemia. While this may be an important first step alerting clinicians that there may be a higher chance for shock development in patients who develop candidemia early on the course of ICU stay, we realize that the limited sample size precludes developing a more powerful model for better prediction of shock.

While not statistically significant, proportionally more patients with $C$ albicans developed shock. This observation was previously reported by Hadley et al, where 7 out of 10 described episodes of candidemia and shock were caused by C. albicans [16]. Although it is tempting to postulate that inherent properties of each species would induce varying degrees of inflammatory response and thus explain the higher incidence of shock observed by C. albicans, this hypothesis was not supported by Wisplinghoff et al [15]. The authors reported similar initial and most severe inflammatory responses with both $C$ albicans and non-albicans Candida spp. over time. The limited sample size precludes reaching any definitive conclusion, although these data may be hypothesis generating and lead to further investigation to determine whether different species trigger different host response. Although there has been a reported increase in the incidence of non-albicans Candida fungemia [6,10], the observed ratio of non-albicans to C. albicans in our cohort is extremely high. It remains unknown whether this is a reflection of antibiotic and antifungal use within our medical center, or a reflection of severity of disease in patients acquiring candidemia. Nonetheless, in selecting the initial early empiric antifungals one should recognize the high frequency of nonalbicans Candida species with intrinsic reduced fluconazole susceptibility.

Our study has limitations. Because of its retrospective nature, the definition of shock induced by candidemia may be disputed, although we only included patients who had hypotension not explained by any other reason than infection and the percentage of patients progressing to shock is similar to previously described [15]. Similarly, colonization was not documented in all patients as it is not routine to do surveillance cultures for Candida in the US. The study period preceded the introduction of echinocandins into clinical use. In spite of these limitations, this is to our knowledge one of the largest cohorts of patients with Candida induced septic shock and the first that has attempted to identify predictors of shock development thus far.

In summary, septic shock due to candidemia in the ICU setting is associated with extremely high mortality. No conventional risk factors were identified to predict shock development other than the time spent in ICU until the development of candidemia. Given the high mortality associated with candidemic septic shock, consideration should be given for initiating empiric broad spectrum cidal antifungal therapy in high risk ICU patients already receiving and not responding to antibiotic therapy. This therapeutic approach should be validated by prospective studies.

\section{References}

1. Edmond MB, Wallace SE, McClish DK, Pfaller MA, Jones RN, Wenzel RP. Nosocomial bloodstream infections in United States hospitals: a three-year analysis. Clin Infect Dis 1999;29(2):239-244.

2. Pfaller MA, Diekema DJ, Jones RN, Sader HS, Fluit AC, Hollis RJ, Messer SA. International surveillance of bloodstream infections due to Candida species: frequency of occurrence and in vitro susceptibilities to fluconazole, ravuconazole, and voriconazole of isolates 
collected from 1997 through 1999 in the SENTRY antimicrobial surveillance program. J Clin Microbiol 2001;39(9):3254-3259.

3. Marchetti O, Bille J, Fluckiger U, Eggimann P, Ruef C, Garbino J, Calandra T, et al. Epidemiology of candidemia in Swiss tertiary care hospitals: secular trends, 1991-2000. Clin Infect Dis 2004;38(3):311-320.

4. Nguyen MH, Peacock JE, Jr., Morris AJ, Tanner DC, Nguyen ML, Snydman DR, Wagener MM, et al. The changing face of candidemia: emergence of non-Candida albicans species and antifungal resistance. Am J Med 1996;100(6):617-623.

5. Eggimann P, Garbino J, Pittet D. Epidemiology of Candida species infections in critically ill non-immunosuppressed patients. Lancet Infect Dis 2003;3(11):685-702.

6. Bassetti M, Righi E, Costa A, Fasce R, Molinari MP, Rosso R, Pallavicini FB, et al. Epidemiological trends in nosocomial candidemia in intensive care. BMC Infect Dis 2006;6:21.

7. Voss A, le Noble JL, Verduyn Lunel FM, Foudraine NA, Meis JF. Candidemia in intensive care unit patients: risk factors for mortality. Infection 1997;25(1):8-11.

8. Komshian SV, Uwaydah AK, Sobel JD, Crane LR. Fungemia caused by Candida species and Torulopsis glabrata in the hospitalized patient: frequency, characteristics, and evaluation of factors influencing outcome. Rev Infect Dis 1989;11(3):379-390.

9. Dimopoulos G, Ntziora F, Rachiotis G, Armaganidis A, Falagas ME. Candida albicans versus non-albicans intensive care unit-acquired bloodstream infections: differences in risk factors and outcome. Anesth Analg 2008;106(2):523-529, table of contents.

10. Blot S, Vandewoude K, Hoste E, Poelaert J, Colardyn F. Outcome in critically ill patients with candidal fungaemia: Candida albicans vs. Candida glabrata. J Hosp Infect 2001;47(4):308-313.

11. Gudlaugsson O, Gillespie S, Lee K, Vande Berg J, Hu J, Messer S, Herwaldt L, et al. Attributable mortality of nosocomial candidemia, revisited. Clin Infect Dis 2003;37(9):1172-1177.

12. Labelle AJ, Micek ST, Roubinian N, Kollef MH. Treatment-related risk factors for hospital mortality in Candida bloodstream infections. Crit Care Med
2008;36(11):2967-2972.

13. Leon C, Ruiz-Santana S, Saavedra P, Almirante B, Nolla-Salas J, Alvarez-Lerma F, Garnacho-Montero J, et al. A bedside scoring system ("Candida score") for early antifungal treatment in nonneutropenic critically ill patients with Candida colonization. Crit Care Med 2006;34(3):730-737.

14. Wenzel RP, Gennings C. Bloodstream infections due to Candida species in the intensive care unit: identifying especially high-risk patients to determine prevention strategies. Clin Infect Dis 2005;41 Suppl 6:S389-393.

15. Wisplinghoff H, Seifert H, Wenzel RP, Edmond MB. Inflammatory response and clinical course of adult patients with nosocomial bloodstream infections caused by Candida spp. Clin Microbiol Infect 2006;12(2):170-177.

16. Hadley S, Lee WW, Ruthazer R, Nasraway SA, Jr. Candidemia as a cause of septic shock and multiple organ failure in nonimmunocompromised patients. Crit Care Med 2002;30(8):1808-1814.

17. Levy MM, Fink MP, Marshall JC, Abraham E, Angus D, Cook D, Cohen J, et al. 2001 SCCM/ESICM/ACCP/ ATS/SIS International Sepsis Definitions Conference. Crit Care Med 2003;31(4):1250-1256.

18. Patel GP, Simon D, Scheetz M, Crank CW, Lodise T, Patel N. The effect of time to antifungal therapy on mortality in Candidemia associated septic shock. Am J Ther 2009;16(6):508-511.

19. Pappas PG, Kauffman CA, Andes D, Benjamin DK, Jr., Calandra TF, Edwards JE, Jr., Filler SG, et al. Clinical practice guidelines for the management of candidiasis: 2009 update by the Infectious Diseases Society of America. Clin Infect Dis 2009;48(5):503-535.

20. Paphitou NI, Ostrosky-Zeichner L, Rex JH. Rules for identifying patients at increased risk for candidal infections in the surgical intensive care unit: approach to developing practical criteria for systematic use in antifungal prophylaxis trials. Med Mycol 2005;43(3):235-243.

21. Ostrosky-Zeichner L, Sable C, Sobel J, Alexander BD, Donowitz G, Kan V, Kauffman CA, et al. Multicenter retrospective development and validation of a clinical prediction rule for nosocomial invasive candidiasis in the intensive care setting. Eur J Clin Microbiol Infect Dis 2007;26(4):271-276. 